\title{
An On-Line Method for Tracking the Rotor Time Constant of an Induction Machine
}

\author{
Kaiyu Wang, John Chiasson, Marc Bodson, and Leon M. Tolbert
}

\begin{abstract}
Field-oriented control methodology requires knowledge of the machine parameters and, in particular, the rotor time constant. The interest here is in tracking the value of $T_{R}$ as it changes due to ohmic heating so that an accurate value is available to estimate the rotor flux linkages for a field-oriented controller. The approach presented here uses a nonlinear least-squares approach using measurements of the stator currents and voltages along with the rotor speed. The nature of this technique lends itself directly to an online implementation and therefore can be used to track the rotor time constant. Experimental results are presented to demonstrate the validity of the approach.
\end{abstract}

Index Terms - Induction Motor, Rotor Time Constant, Parameter Identification, Resultants

\section{INTRODUCTION}

The field-oriented control method provides a means to obtain high performance control of an induction machine for use in applications such as traction drives. This fieldoriented control methodology requires knowledge of the machine parameters, and in particular the rotor time constant which can vary due to Ohmic heating. The problem is further complicated by the fact that rotor variables are not usually available for measurement. The induction motor parameters are $M$ (the mutual inductance), $L_{S}, L_{R}$ (the stator and rotor inductances), $R_{S}, R_{R}$ (the stator and rotor resistances), $J$ (the inertia of the rotor) which are required for field-oriented control.

Standard methods for the estimation of induction motor parameters include the locked rotor test, the no-load test, and the standstill frequency response test. However, these approaches cannot be used online, that is, during normal operation of the machine. For example, field-oriented control requires knowledge of the rotor time constant $T_{R}=$ $L_{R} / R_{R}$, which varies significantly due to ohmic heating to estimate the rotor flux linkages. The interest here is in tracking the value of $T_{R}$ as it changes due to ohmic heating. The approach presented here is a nonlinear leastsquares method using measurements of the stator currents

K. Wang, J. Chiasson, and L. M. Tolbert are with the ECE Department, University of Tennessee, Knoxville, TN 37996. wkaiyu@utk.edu, chiasson@utk.edu, tolbert@utk.edu.

M. Bodson is with the ECE Department, University of Utah, Salt Lake City, UT 84112 bodson@ece.utah.edu

L. M. Tolbert is also with Oak Ridge National Laboratory, Oak Ridge TN. tolbertlm@ornl.gov

Drs. Chiasson and Tolbert would like to thank Oak Ridge National Laboratory for partially supporting this work through the UT/Battelle contract no. 4000007596. Dr. Tolbert would also like to thank the National Science Foundation for partially supporting this work through contract NSF ECS-0093884. and voltages along with the rotor speed. Due to the nature of this technique, it lends itself directly to an online implementation and therefore can be used to track the rotor time constant.

It turns out that, as the rotor state variables are not available measurements, the system identification model cannot be made linear in the parameters without overparameterizing the model. Here the model is reformulated so that it is a nonlinear system identification problem that is not overparameterized. Further, a method is presented that guarantees the solution for the parameter vector that minimizes the least-squares error. This proposed method improves upon the linear least-squares approach formulated in [1][2]. The work in [1][2] was limited in that the acceleration was required to be small and that the iterative method used to solve the least squares problem was not guaranteed to converge nor necessarily achieve the minimum.

Here, elimination theory [3][4] is used to solve the nonlinear least squares problem, which in turn guarantees the minimum is found with no assumptions on the machine's speed or acceleration; the data need only be sufficiently rich as described in the paper. Experimental results are presented to demonstrate the validity of the approach.

A combined parameter identification and velocity estimation problem is discussed in [5][6][7]. The velocity estimation problem is not considered, but the velocity is allowed to vary. For a summary of the various techniques for tracking the rotor time constant, the reader is referred to the recent survey [8], the recent paper [9], and to the book [10].

The paper is organized as follows. Section II introduces a standard induction motor model expressed in the rotor coordinates. Then, an overparameterized model which is linear in the unknown parameters is derived and discussed in Section III. Section IV presents the identification scheme for the induction motor. Specifically, an overparameterized linear model is reduced to an nonlinear model which is not overparameterized. An approach to solve the resulting nonlinear least-squares identification problem for the rotor time constant is presented which guarantees the solution is found in a finite number of steps. Section V presents the results of the identification algorithm with experimental data.

\section{INDUCTION MOTOR MODEL}

Standard models of induction machines are available in the literature. Parasitic effects such as hysteresis, eddy 
currents, magnetic saturation, and others are generally neglected. Consider the state space model of the system given by (cf. [11][12])

$$
\begin{aligned}
\frac{d \omega}{d t} & =\frac{M n_{p}}{J L_{R}}\left(i_{S b} \psi_{R a}-i_{S a} \psi_{R b}\right)-\frac{\tau_{L}}{J} \\
\frac{d \psi_{R a}}{d t} & =-\frac{1}{T_{R}} \psi_{R a}-n_{p} \omega \psi_{R b}+\frac{M}{T_{R}} i_{S a} \\
\frac{d \psi_{R b}}{d t} & =-\frac{1}{T_{R}} \psi_{R b}+n_{p} \omega \psi_{R a}+\frac{M}{T_{R}} i_{S b} \\
\frac{d i_{S a}}{d t} & =\frac{\beta}{T_{R}} \psi_{R a}+\beta n_{p} \omega \psi_{R b}-\gamma i_{S a}+\frac{1}{\sigma L_{S}} u_{S a} \\
\frac{d i_{S b}}{d t} & =\frac{\beta}{T_{R}} \psi_{R b}-\beta n_{p} \omega \psi_{R a}-\gamma i_{S b}+\frac{1}{\sigma L_{S}} u_{S b}
\end{aligned}
$$

where $\omega=d \theta / d t$ with $\theta$ the position of the rotor, $n_{p}$ is the number of pole pairs, $i_{S a}, i_{S b}$ are the (two phase equivalent) stator currents, and $\psi_{R a}, \psi_{R b}$ are the (two phase equivalent) rotor fluxes.

The parameters of the model are the five electrical parameters, $R_{S}$ and $R_{R}$ (the stator and rotor resistances), $M$ (the mutual inductance), $L_{S}$ and $L_{R}$ (the stator and rotor inductances), and the two mechanical parameters, $J$ (the inertia of the rotor) and $\tau_{L}$ (the load torque). The symbols

$$
\begin{array}{ll}
T_{R}=\frac{L_{R}}{R_{R}} & \sigma=1-\frac{M^{2}}{L_{S} L_{R}} \\
\beta=\frac{M}{\sigma L_{S} L_{R}} & \gamma=\frac{R_{S}}{\sigma L_{S}}+\frac{M^{2} R_{R}}{\sigma L_{S} L_{R}^{2}}
\end{array}
$$

have been used to simplify the expressions. $T_{R}$ is referred to as the rotor time constant while $\sigma$ is called the total leakage factor.

This model is transformed into a coordinate system attached to the rotor. For example, the current variables are transformed according to

$$
\left[\begin{array}{l}
i_{S x} \\
i_{S y}
\end{array}\right]=\left[\begin{array}{rr}
\cos \left(n_{p} \theta\right) & \sin \left(n_{p} \theta\right) \\
-\sin \left(n_{p} \theta\right) & \cos \left(n_{p} \theta\right)
\end{array}\right]\left[\begin{array}{c}
i_{S a} \\
i_{S b}
\end{array}\right] .
$$

The transformation simply projects the vectors in the $(a, b)$ frame onto the axes of the moving coordinate frame. An advantage of this transformation is that the signals in the moving frame (i.e., the $(x, y)$ frame) typically vary slower than those in the $(a, b)$ frame (they vary at the slip frequency rather than at the stator frequency). At the same time, the transformation does not depend on any unknown parameter in contrast to the field-oriented transformation. The stator voltages and the rotor flux linkages are transformed as the currents resulting in the following model ([2])

$$
\begin{aligned}
\frac{d i_{S x}}{d t}= & \frac{u_{S x}}{\sigma L_{S}}-\gamma i_{S x}+\frac{\beta}{T_{R}} \psi_{R x}+n_{p} \beta \omega \psi_{R y} \\
& +n_{p} \omega i_{S y} \\
\frac{d i_{S y}}{d t}= & \frac{u_{S y}}{\sigma L_{S}}-\gamma i_{S y}+\frac{\beta}{T_{R}} \psi_{R y}-n_{p} \beta \omega \psi_{R x} \\
& -n_{p} \omega i_{S x} \\
\frac{d \psi_{R x}}{d t}= & \frac{M}{T_{R}} i_{S x}-\frac{1}{T_{R}} \psi_{R x} \\
\frac{d \psi_{R y}}{d t}= & \frac{M}{T_{R}} i_{S y}-\frac{1}{T_{R}} \psi_{R y} \\
\frac{d \omega}{d t}= & \frac{M n_{p}}{J L_{R}}\left(i_{S y} \psi_{R x}-i_{S x} \psi_{R y}\right)-\frac{\tau_{L}}{J} .
\end{aligned}
$$

\section{LINEAR OVERPARAMETERIZED MODEL}

As stated in the introduction, the interest here is in tracking the value of $T_{R}$ as it changes due to ohmic heating so that an accurate value is available to estimate the rotor flux linkages for a field oriented controller. However, the stator resistance value $R_{S}$ will also vary due to ohmic heating so that it must also be taken into account. The electrical parameters $M, L_{S}, \sigma$ are assumed to be known and not varying. Measurements of the stator currents $i_{S a}, i_{S b}$ and voltages $u_{S a}, u_{S b}$ as well as the position $\theta$ of the rotor are assumed to be available; the velocity is then reconstructed from the position measurements. However, the rotor flux linkages are not assumed to be measured.

Standard methods for parameter estimation are based on equalities where known signals depend linearly on unknown parameters. However, the induction motor model described above does not fit in this category unless the rotor flux linkages are measured. The first step is to eliminate the fluxes $\psi_{R x}, \psi_{R y}$ and their derivatives $d \psi_{R x} / d t, d \psi_{R y} / d t$ from the model. The four equations (3), (4), (5), (6) can be used to solve for $\psi_{R x}, \psi_{R y}, d \psi_{R x} / d t, d \psi_{R y} / d t$, but one is left without another independent equation to set up a regressor system for the identification algorithm. A new set of independent equations are found by differentiating equations (3) and (4) to obtain

$$
\begin{aligned}
\frac{1}{\sigma L_{s}} \frac{d u_{S x}}{d t}= & \frac{d^{2} i_{S x}}{d t^{2}}+\gamma \frac{d i_{S x}}{d t}-\frac{\beta}{T_{R}} \frac{d \psi_{R x}}{d t}-n_{p} \beta \omega \frac{d \psi_{R y}}{d t} \\
& -n_{p} \beta \psi_{R y} \frac{d \omega}{d t}-n_{p} \omega \frac{d i_{S y}}{d t}-n_{p} i_{S y} \frac{d \omega}{d t}
\end{aligned}
$$

and

$$
\begin{aligned}
\frac{1}{\sigma L_{s}} \frac{d u_{S y}}{d t}= & \frac{d^{2} i_{S y}}{d t^{2}}+\gamma \frac{d i_{S y}}{d t}-\frac{\beta}{T_{R}} \frac{d \psi_{R y}}{d t}+n_{p} \beta \omega \frac{d \psi_{R x}}{d t} \\
& +n_{p} \beta \psi_{R x} \frac{d \omega}{d t}+n_{p} \omega \frac{d i_{S x}}{d t}+n_{p} i_{S x} \frac{d \omega}{d t} .
\end{aligned}
$$

Next, equations (3), (4), (5), (6) are solved for $\psi_{R x}, \psi_{R y}$, $d \psi_{R x} / d t, d \psi_{R y} / d t$ and substituted into equations (8) and (9) 
to obtain

$$
\begin{aligned}
0 & =-\frac{d^{2} i_{S x}}{d t^{2}}+\frac{d i_{S y}}{d t} n_{p} \omega+\frac{1}{\sigma L_{S}} \frac{d u_{S x}}{d t}-\left(\gamma+\frac{1}{T_{R}}\right) \frac{d i_{S x}}{d t} \\
& -i_{S x}\left(-\frac{\beta M}{T_{R}^{2}}+\frac{\gamma}{T_{R}}\right)+i_{S y} n_{p} \omega\left(\frac{1}{T_{R}}+\frac{\beta M}{T_{R}}\right)+\frac{u_{S x}}{\sigma L_{S} T_{R}} \\
& +n_{p} \frac{d \omega}{d t} i_{S y}-n_{p} \frac{d \omega}{d t} \frac{1}{\sigma L_{S}\left(1+n_{p}^{2} \omega^{2} T_{R}^{2}\right)} \times \\
& \left(-\sigma L_{S} T_{R} \frac{d i_{S y}}{d t}-\gamma i_{S y} \sigma L_{S} T_{R}-i_{S x} n_{p} \omega \sigma L_{S} T_{R}\right. \\
& -\frac{d i_{S x}}{d t} n_{p} \omega \sigma L_{S} T_{R}^{2}-\gamma i_{S x} n_{p} \omega \sigma L_{S} T_{R}^{2}+i_{S y} n_{p}^{2} \omega^{2} \sigma L_{S} T_{R}^{2} \\
& \left.+n_{p} \omega T_{R}^{2} u_{S x}+T_{R} u_{S y}\right)
\end{aligned}
$$

$0=-\frac{d^{2} i_{S y}}{d t^{2}}-\frac{d i_{S x}}{d t} n_{p} \omega+\frac{1}{\sigma L_{S}} \frac{d u_{S y}}{d t}-\left(\gamma+\frac{1}{T_{R}}\right) \frac{d i_{S y}}{d t}$

$$
-i_{S y}\left(-\frac{\beta M}{T_{R}^{2}}+\frac{\gamma}{T_{R}}\right)-i_{S x} n_{p} \omega\left(\frac{1}{T_{R}}+\frac{\beta M}{T_{R}}\right)+\frac{u_{S y}}{\sigma L_{S} T_{R}}
$$$$
-n_{p} \frac{d \omega}{d t} i_{S x}+n_{p} \frac{d \omega}{d t} \frac{1}{\sigma L_{S}\left(1+n_{p}^{2} \omega^{2} T_{R}^{2}\right)} \times
$$$$
\left(-\sigma L_{S} T_{R} \frac{d i_{S x}}{d t}-\gamma i_{S x} \sigma L_{S} T_{R}+i_{S y} n_{p} \omega \sigma L_{S} T_{R}\right.
$$$$
+\frac{d i_{S y}}{d t} n_{p} \omega \sigma L_{S} T_{R}^{2}+\gamma i_{S y} n_{p} \omega \sigma L_{S} T_{R}^{2}+i_{S x} n_{p}^{2} \omega^{2} \sigma L_{S} T_{R}^{2}
$$$$
\left.-n_{p} \omega T_{R}^{2} u_{S y}+T_{R} u_{S x}\right) \text {. }
$$

This set of equations may be rewritten in regressor form as

$$
y(t)=W(t) K
$$

where $W \in \mathbb{R}^{2 \times 8}, K \in \mathbb{R}^{8}$ and $y \in \mathbb{R}^{2}$ are given by

$$
\begin{gathered}
W=\left[\begin{array}{cc}
-\frac{d i_{S x}}{d t}-\frac{d i_{S x}}{d t}+n_{p} \omega i_{S y}+n_{p} \omega M \beta i_{S y}+\frac{u_{S x}}{\sigma L s} \\
-\frac{d i_{S y}}{d t}-\frac{d i_{S y}}{d t}-n_{p} \omega i_{S x}-n_{p} \omega M \beta i_{S x}+\frac{u_{S y}}{\sigma L s}
\end{array}\right. \\
M \beta i_{S x}-i_{S x} \quad n_{p} \frac{d i_{S y}}{d t} \frac{d \omega}{d t}+n_{p}^{2}\left(\omega i_{S x} \frac{d \omega}{d t}-\omega^{2} \frac{d i_{S x}}{d t}\right) \\
M \beta i_{S y}-i_{S y}-n_{p} \frac{d i_{S x}}{d t} \frac{d \omega}{d t}+n_{p}^{2}\left(\omega i_{S y} \frac{d \omega}{d t}-\omega^{2} \frac{d i_{S y}}{d t}\right) \\
+n_{p}^{3} \omega^{3} i_{S y}(1+M \beta)+\frac{1}{\sigma L s}\left(n_{p}^{2} \omega^{2} u_{S x}-n_{p} u_{S y} \frac{d \omega}{d t}\right) \\
-n_{p}^{3} \omega^{3} i_{S x}(1+M \beta)+\frac{1}{\sigma L s}\left(n_{p}^{2} \omega^{2} u_{S y}+n_{p} u_{S x} \frac{d \omega}{d t}\right)
\end{gathered}
$$

$$
\begin{aligned}
& n_{p} i_{S y} \frac{d \omega}{d t}-n_{p}^{2} \omega^{2} i_{S x} \quad n_{p}^{2}\left(i_{s x} \omega \frac{d \omega}{d t}-\omega^{2} \frac{d i_{S x}}{d t}\right) \\
& -n_{p} i_{S x} \frac{d \omega}{d t}-n_{p}^{2} \omega^{2} i_{S y} \quad n_{p}^{2}\left(i_{s y} \omega \frac{d \omega}{d t}-\omega^{2} \frac{d i_{S y}}{d t}\right) \\
& n_{p}^{2}\left(\omega \frac{d i_{S x}}{d t} \frac{d \omega}{d t}-\omega^{2} \frac{d^{2} i_{S x}}{d t^{2}}\right)+\frac{d i_{S y}}{d t} n_{p}^{3} \omega^{3} \\
& n_{p}^{2}\left(\omega \frac{d i_{S y}}{d t} \frac{d \omega}{d t}-\omega^{2} \frac{d^{2} i_{S y}}{d t^{2}}\right)-\frac{d i_{S x}}{d t} n_{p}^{3} \omega^{3} \\
& -\frac{n_{p}^{2}}{\sigma L s}\left(\omega u_{S x} \frac{d \omega}{d t}-\omega^{2} \frac{d u_{S x}}{d t}\right) \\
& \left.-\frac{n_{p}^{2}}{\sigma L s}\left(\omega u_{S y} \frac{d \omega}{d t}-\omega^{2} \frac{d u_{S y}}{d t}\right)\right] \\
& K \triangleq\left[\begin{array}{llllllll}
\gamma & \frac{1}{T_{R}} & \frac{1}{T_{R}^{2}} & \frac{\gamma}{T_{R}} & T_{R} & \gamma T_{R} & \gamma T_{R}^{2} & T_{R}^{2}
\end{array}\right]^{T} \\
& \text { and } \\
& y \triangleq\left[\begin{array}{l}
\frac{d^{2} i_{S x}}{d t^{2}}-n_{p} i_{S y} \frac{d \omega}{d t}-n_{p} \omega \frac{d i_{S y}}{d t}-n_{p}^{2} \omega^{2} M \beta i_{S x} \\
\frac{d^{2} i_{S y}}{d t^{2}}+n_{p} i_{S x} \frac{d \omega}{d t}+n_{p} \omega \frac{d i_{S x}}{d t}-n_{p}^{2} \omega^{2} M \beta i_{S y}
\end{array}\right. \\
& \left.\begin{array}{l}
-\frac{1}{\sigma L s} \frac{d u_{S x}}{d t} \\
-\frac{1}{\sigma L s} \frac{d v_{S y}}{d t}
\end{array}\right]
\end{aligned}
$$

As $M^{2} / L_{R}=(1-\sigma) L_{S}, M \beta=(1-\sigma) / \sigma, \gamma=\frac{R_{S}}{\sigma L_{S}}+$ $\frac{1}{\sigma L_{S}} \frac{1}{T_{R}}(1-\sigma) L_{S}$ it is seen that $y$ and $W$ depend only on known quantities while the unknowns $R_{S}, T_{R}$ are contained only within $K$.

Though the system regressor is linear in the parameters, one cannot use standard least-squares techniques as the system is overparameterized. Specifically,

$$
\begin{aligned}
& K_{3}=K_{2}^{2}, K_{4}=K_{1} K_{2}, K_{5}=1 / K_{2}, K_{6}=K_{1} / K_{2}, \\
& K_{7}=K_{1} / K_{2}^{2}, K_{8}=1 / K_{2}^{2}
\end{aligned}
$$

so that only the two parameters $K_{1}, K_{2}$ are independent. These two parameters determine $R_{S}$ and $T_{R}$ by

$$
\begin{aligned}
& T_{R}=1 / K_{2} \\
& R_{S}=\sigma L_{S} K_{1}-(1-\sigma) L_{S} K_{2} . \\
& \text { IV. NONLINEAR LEAST-SQUARES } \\
& \text { IDENTIFICATION[13][14][15] }
\end{aligned}
$$

With an abuse of notation, equation (12) can be rewritten as

$$
y(n)=W(n) K
$$

where $n$ is the time instant at which a measurement is taken and $K$ is the vector of unknown parameters. However, this 
is an overparameterized representation of the model. Also, several factors contribute to errors which make equation (16) only approximately valid in practice. Specifically, both $y(n)$ and $W(n)$ are measured through signals that are noisy (in particular due to quantization and differentiation) and the mathematical model of the induction motor is only an approximate representation of the real system. These sources of error result in an inconsistent system of equations. To find a solution for such a system, the least-squares criterion is still used. Specifically, given $y(n)$ and $W(n)$ and a parameter vector $K$, the squared-error is defined by

$$
\begin{aligned}
E^{2}(K) & \triangleq \sum_{n=1}^{N}|y(n)-W(n) K|^{2} \\
& =R_{y}-2 R_{W y}^{T} K+K^{T} R_{W} K
\end{aligned}
$$

where

$$
\begin{aligned}
R_{W} & \triangleq \sum_{n=1}^{N} W(n) W^{T}(n) \\
R_{W y} & \triangleq \sum_{n=1}^{N} W(n) y(n) \\
R_{y} & \triangleq \sum_{n=1}^{N} y^{T}(n) y(n) .
\end{aligned}
$$

The nonlinear least-squares criterion requires minimizing (17) subject to the constraints (14). On physical grounds, the parameters $K_{1}, K_{2}$ are constrained to

$$
0<K_{1}<\infty, 0<K_{2}<\infty .
$$

Also, based on physical grounds, the squared error $E^{2}(K)$ will be minimized in the interior of this region. Let

$$
\begin{aligned}
& E^{2}\left(K_{p}\right) \triangleq \sum_{n=1}^{N}|y(n)-W(n) K|_{\substack{K_{3}=K_{2}^{2} \\
K_{4}=K_{1} K_{2}}}^{2} \\
& =R_{y}-\left.2 R_{W y}^{T} K\right|_{\substack{K_{3}=K_{2}^{2} \\
K_{4}=K_{1} K_{2}}}+\stackrel{\vdots}{\left.K^{T} R_{W} K\right)\left.\right|_{\substack{K_{3}=K_{2}^{2} \\
K_{4}=K_{1} K_{2}}}}
\end{aligned}
$$

where

$$
K_{p} \triangleq\left[\begin{array}{ll}
K_{1} & K_{2}
\end{array}\right]^{T}
$$

As just explained, the minimum of (19) must occur in the interior of the region and therefore at an extremum point. This then entails solving the two equations

$$
\begin{aligned}
& r_{1}\left(K_{p}\right) \triangleq \frac{\partial E^{2}\left(K_{p}\right)}{\partial K_{1}}=0 \\
& r_{2}\left(K_{p}\right) \triangleq \frac{\partial E^{2}\left(K_{p}\right)}{\partial K_{2}}=0 .
\end{aligned}
$$

The partial derivatives in (20)-(21) are rational functions in the parameters $K_{1}, K_{2}$. Defining

$$
\begin{aligned}
& p_{1}\left(K_{p}\right) \triangleq K_{2}^{4} r_{1}\left(K_{p}\right)=K_{2}^{4} \frac{\partial E^{2}\left(K_{p}\right)}{\partial K_{1}} \\
& p_{2}\left(K_{p}\right) \triangleq K_{2}^{5} r_{2}\left(K_{p}\right)=K_{2}^{5} \frac{\partial E^{2}\left(K_{p}\right)}{\partial K_{2}}
\end{aligned}
$$

results in the $p_{i}\left(K_{p}\right)$ being polynomials in the parameters $K_{1}, K_{2}$ and having the same positive zero set (i.e., the same roots satisfying $K_{i}>0$ ) as the system (20)-(21). The degrees of the polynomials $p_{i}$ are given in the table below.

\begin{tabular}{|c|c|c|}
\hline & $\operatorname{deg} K_{1}$ & $\operatorname{deg} K_{2}$ \\
\hline$p_{1}\left(K_{p}\right)$ & 1 & 7 \\
\hline$p_{2}\left(K_{p}\right)$ & 2 & 8 \\
\hline
\end{tabular}

All possible solutions to this set may be found using elimination theory as is now summarized.

\section{A. Solving Systems of Polynomial Equations [3][4]}

The question at hand is "Given two polynomial equations $a\left(K_{1}, K_{2}\right)=0$ and $b\left(K_{1}, K_{2}\right)=0$, how does one solve them simultaneously to eliminate (say) $K_{2}$ ?". A systematic procedure to do this is known as elimination theory and uses the notion of resultants. Briefly, one considers $a\left(K_{1}, K_{2}\right)$ and $b\left(K_{1}, K_{2}\right)$ as polynomials in $K_{2}$ whose coefficients are polynomials in $K_{1}$. Then, for example, letting $a\left(K_{1}, K_{2}\right)$ and $b\left(K_{1}, K_{2}\right)$ have degrees 3 and 2 , respectively in $K_{2}$, they may be written in the form

$$
\begin{aligned}
a\left(K_{1}, K_{2}\right)= & a_{3}\left(K_{1}\right) K_{2}^{3}+a_{2}\left(K_{1}\right) K_{2}^{2}+a_{1}\left(K_{1}\right) K_{2} \\
& +a_{0}\left(K_{1}\right) \\
b\left(K_{1}, K_{2}\right)= & b_{2}\left(K_{1}\right) K_{2}^{2}+b_{1}\left(K_{1}\right) K_{2}+b_{0}\left(K_{1}\right) .
\end{aligned}
$$

The $n \times n$ Sylvester matrix, where $n=$ $\operatorname{deg}_{K_{2}}\left\{a\left(K_{1}, K_{2}\right)\right\}+\operatorname{deg}_{K_{2}}\left\{b\left(K_{1}, K_{2}\right)\right\}=3+2=5$, is defined by

$$
\begin{aligned}
& S_{a, b}\left(K_{1}\right)= \\
& {\left[\begin{array}{ccccc}
a_{0}\left(K_{1}\right) & 0 & b_{0}\left(K_{1}\right) & 0 & 0 \\
a_{1}\left(K_{1}\right) & a_{0}\left(K_{1}\right) & b_{1}\left(K_{1}\right) & b_{0}\left(K_{1}\right) & 0 \\
a_{2}\left(K_{1}\right) & a_{1}\left(K_{1}\right) & b_{2}\left(K_{1}\right) & b_{1}\left(K_{1}\right) & b_{0}\left(K_{1}\right) \\
a_{3}\left(K_{1}\right) & a_{2}\left(K_{1}\right) & 0 & b_{2}\left(K_{1}\right) & b_{1}\left(K_{1}\right) \\
0 & a_{3}\left(K_{1}\right) & 0 & 0 & b_{2}\left(K_{1}\right)
\end{array}\right] .}
\end{aligned}
$$

The resultant polynomial is then defined by

$$
r\left(K_{1}\right)=\operatorname{Res}\left(a\left(K_{1}, K_{2}\right), b\left(K_{1}, K_{2}\right), K_{2}\right) \triangleq \operatorname{det} S_{a, b}\left(K_{1}\right)
$$

and is the result of eliminating the variable $K_{2}$ from $a\left(K_{1}, K_{2}\right)$ and $b\left(K_{1}, K_{2}\right)$. In fact, the following is true.

Theorem 1: Any solution $\left(K_{10}, K_{20}\right)$ of $a\left(K_{1}, K_{2}\right)=0$ and $b\left(K_{1}, K_{2}\right)=0$ must have $r\left(K_{10}\right)=0$. [3][4].

Though the converse of this theorem is not necessarily true, the finite number of solutions of $r\left(K_{1}\right)=0$ are the only possible candidates for the first coordinate (partial solutions) of the common zeros of $a\left(K_{1}, K_{2}\right)$ and $b\left(K_{1}, K_{2}\right)$. Whether or not such a partial solution results in a full solution is simply determined by back solving and checking the solution. 


\section{B. Solving the Nonlinear Least-squares Problem}

Using the polynomials (22)-(23), the variable $K_{1}$ is eliminated to obtain

$$
r\left(K_{2}\right) \triangleq \operatorname{Res}\left(p_{1}\left(K_{1}, K_{2}\right), p_{2}\left(K_{1}, K_{2}\right), K_{1}\right)
$$

where $\operatorname{deg}_{K_{2}}\left\{r\left(K_{2}\right)\right\}=20$. The parameter $K_{2}$ was chosen as the variable not eliminated because its degree is much higher than $K_{1}$ meaning it would have a larger (in dimension) Sylvester matrix. The positive roots of $r\left(K_{2}\right)=0$ are found which are then substituted into $p_{1}=0$ (or $\left.p_{2}=0\right)$ to find the positive roots in $K_{1}$, etc. By this method of back solving, all possible (finite number) candidate solutions are found and one simply chooses the one that gives the smallest squared error.

\section{Sufficiency of Excitation and Numerical Conditioning}

After finding the solution that gives the minimal value for $E^{2}\left(K_{p}\right)$, one needs to know if the solution makes sense. For example, in the linear least-squares problem, there is a unique well defined solution provided that the regressor matrix $R_{W}$ is nonsingular and its condition number is not too large. In the nonlinear case here, a Taylor series expansion about the computed minimum point $K_{p}^{*}=\left[K_{1}^{*}, K_{2}^{*}\right]^{T}$ gives $(i, j=1,2)$

$$
\begin{aligned}
& E^{2}\left(K_{p}\right)= \\
& \quad E^{2}\left(K_{p}^{*}\right)+\frac{1}{2}\left[K_{p}-K_{p}^{*}\right]^{T} \frac{\partial^{2} E^{2}\left(K_{p}^{*}\right)}{\partial K_{i} \partial K_{j}}\left[K_{p}-K_{p}^{*}\right]+\cdots .
\end{aligned}
$$

One then checks that the Hessian matrix $\frac{\partial^{2} E^{2}\left(K_{p}^{*}\right)}{\partial K_{i} \partial K_{i}}$ is positive definite to ensure that the data is sufficiently rich to identify the parameters as well as its condition number to check the numerical sensitivity of $K_{p}^{*}$ to the data set.

\section{EXPERIMENTAL RESUlTS}

A three-phase, $230 \mathrm{~V}, 0.5 \mathrm{Hp}, 1735 \mathrm{rpm}\left(n_{p}=2\right.$ polepair) induction machine was used for the experiments. A 4096 pulse/rev optical encoder was attached to the motor for position measurements. The motor was connected to a threephase $60 \mathrm{~Hz}$ source through a switch. When the switch was closed, the stator currents and voltages along with the rotor position were sampled at $4 \mathrm{kHz}$. Filtered differentiation (using digital filters) was used for calculating the acceleration and the derivatives of the voltages and currents. Specifically, the signals were filtered with a lowpass digital Butterworth filter followed by reconstruction of the derivatives using $d x(t) / d t=(x(t)-x(t-T)) / T$ where $T$ is the sampling interval. The voltages and currents were put through a 3 to 2 transformation to obtain the two-phase equivalent voltages $u_{S a}, u_{S b}$ which are plotted in Figure 1.

The sampled two-phase equivalent current $i_{S a}$ and it simulated response $i_{S a_{\text {s sim }}}$ are shown in Figure 2 (The simulated current will be discussed below). The phase $b$ current $i_{S b}$ is similar, but shifted by $\pi /\left(2 n_{p}\right)$.

The calculated speed $\omega$ (from the position measurements) and the simulated speed $\omega_{\text {sim }}$ are shown in Figure 3 (the

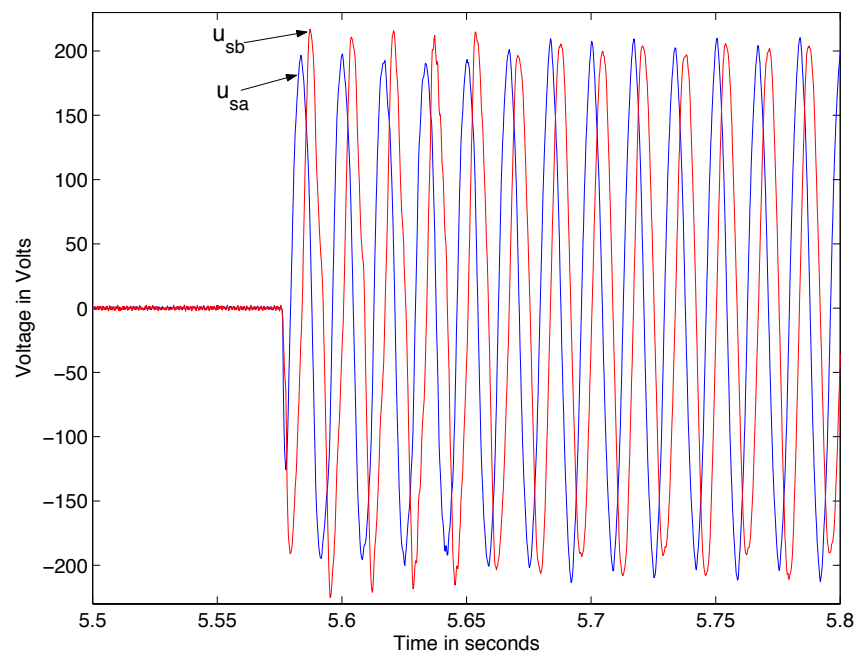

Fig. 1. Sampled two-phase equivalent voltages $u_{S a}$ and $u_{S b}$.

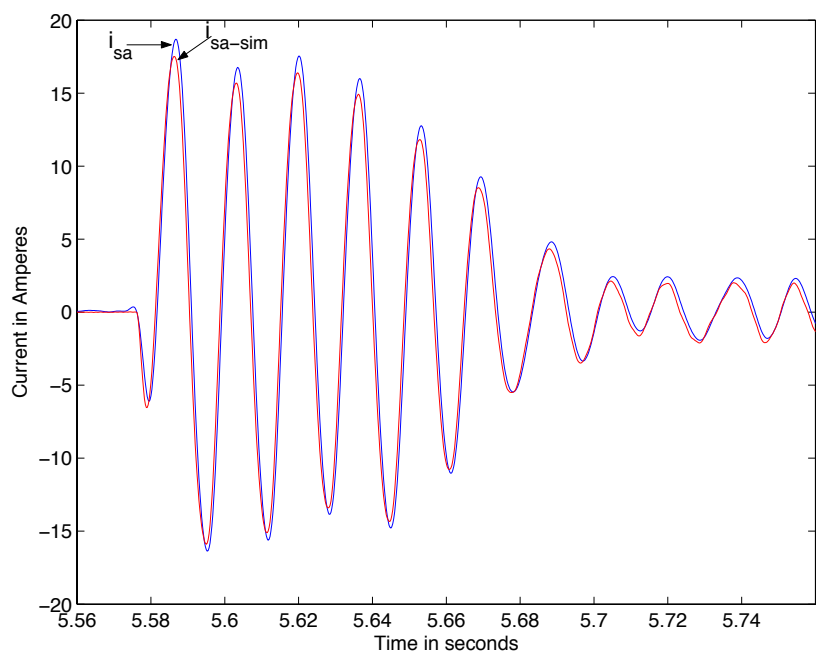

Fig. 2. Phase a current $i_{S a}$ and its simulated response $i_{S a}$ sim.

simulated speed $\omega_{\text {sim }}$ will be discussed below). Using the data $\left\{u_{S a}, u_{S b}, i_{S a}, i_{S b}, \theta\right\}$ collected between $5.57 \mathrm{sec}$ to $5.8 \mathrm{sec}$, the quantities $u_{S x}, u_{S y}, d u_{S x} / d t, d u_{S y} / d t, i_{S x}, i_{S y}$ $d i_{S x} / d t, d i_{S y} / d t, d^{2} i_{S x} / d t^{2}, d^{2} i_{S y} / d t^{2}, \omega=d \theta / d t, d \omega / d t$ were calculated and the regressor matrices $R_{W}, R_{y}$ and $R_{W y}$ were computed. The procedure explained in Section IV was then carried out to compute $K_{1}, K_{2}$. In this case, there were three extrema points that had positive values for $K_{1}$ and $K_{2}$. The parameter values that resulted in the minimum leastsquares error are 


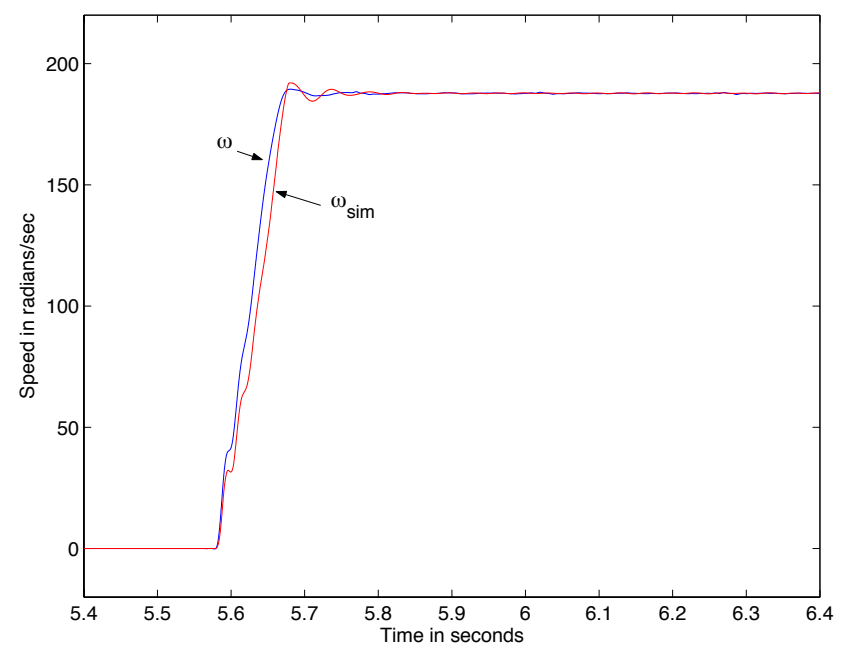

Fig. 3. Calculated speed $\omega$ and simulated speed $\omega_{\text {sim }}$.

Using (15), it follows that

$$
\begin{aligned}
& T_{R}=0.1316 \mathrm{sec} \\
& R_{S}=5.0923 \Omega .
\end{aligned}
$$

By way of comparison, the stator resistance was measured using an Ohmmeter giving the value of $4.9 \mathrm{Ohms}$. The Hessian matrix was calculated at the minimum point according to (25) resulting in

$$
\left\{\frac{\partial^{2} E^{2}\left(K_{p}^{*}\right)}{\partial K_{i} \partial K_{j}}\right\}=\left[\begin{array}{cc}
1.9123 & 0.00412 \\
0.00412 & 570.0418
\end{array}\right]
$$

which is positive definite and has a condition number of $2.98 \times 10^{2}$.

\section{A. Simulation of the Experimental Motor}

Another useful way to evaluate the identified parameters (26) and (27) is to simulate the motor using these values and the measured voltages as input. The model (1) is now in terms of the parameters that can be estimated. The experimental voltages shown in Figure 1 were then used as input to a simulation of the model (1) using the parameter values from (26) and (27). The resulting phase $a$ current $i_{S a \_ \text {sim }}$ from the simulation is shown in Figure 2 and corresponds well with the actual measured current $i_{S a}$. Similarly, the resulting speed $\omega_{\text {sim }}$ from the simulation is shown in Figure 3 where it is seen that the simulated speed is somewhat more oscillatory than the measured speed $\omega$.

\section{CONCLUSIONS}

In this paper, a method for estimating the rotor time constant and stator resistance of an induction machine was presented. The parameter model was formulated as a nonlinear least-squares formulation and then solved using elimination theory. Experimental results showed a close correlation with simulations based on the identified parameters. An important advantage of the procedure is that it can be used online, i.e., during regular operation of the machine, its parameter values can be continuously updated assuming sufficient excitation of the machine.

\section{REFERENCES}

[1] J. Stephan, M. Bodson, and J. Chiasson, "Real-time estimation of induction motor parameters", IEEE Transactions on Industry Applications, vol. 30, no. 3, pp. 746-759, May/June 1994.

[2] J. Stephan, "Real-time estimation of the parameters and fluxes of induction motors", Master's thesis, Carnegie Mellon University, 1992.

[3] David Cox, John Little, and Donal O'Shea, IDEALS, VARIETIES, AND ALGORITHMS An Introduction to Computational Algebraic Geometry and Commutative Algebra, 2nd Edition, Springer-Verlag, Berlin, 1996.

[4] Joachim von zur Gathen and Jürgen Gerhard, Modern Computer Algebra, Cambridge University Press, Cambridge, UK, 1999.

[5] M. Vélez-Reyes, K. Minami, and G. Verghese, "Recursive speed and parameter estimation for induction machines", in Proceedings of the IEEE Industry Applications Conference, 1989, pp. 607-611, San Diego, California.

[6] Miguel Vélez-Reyes, W. L. Fung, and J. E. Ramos-Torres, "Developing robust algorithms for speed and parameter estimation in induction machines", in Proceedings of the IEEE Conference on Decision and Control, 2001, pp. 2223-2228, Orlando, Florida.

[7] Miguel Vélez-Reyes and George Verghese, "Decomposed algorithms for speed and parameter estimation in induction machines", in Proceedings of the IFAC Nonlinear Control Systems Design Symposium, 1992, pp. 156-161, Bordeaux, France.

[8] Hamid A. Toliyat, Emil Levi, and Mona Raina, "A review of RFO induction motor parameter estimation techniques", IEEE Transactions on Energy Conversion, vol. 18, no. 2, pp. 271-283, June 2003.

[9] Miguel Vélez-Reyes, Milan Mijalković, Aleksandar M. Stanković, Silva Hiti, and James Nagashima, "Output selection for tuning of field-oriented controllers: Steady-state analysis", in Conference Record of Industry Applications Society, October 2003, pp. 20122016, Salt Lake City, UT.

[10] Peter Vas, Parameter estimation, condition monitoring, and diagnosis of electrical machines, Oxford: Clarendon Press, 1993.

[11] R. Marino, S. Peresada, and P. Valigi, "Adaptive input-output linearizing control of induction motors", IEEE Transactions on Automatic Control, vol. 38, no. 2, pp. 208-221, February 1993.

[12] M. Bodson, J. Chiasson, and R. Novotnak, "High performance induction motor control via input-output linearization", IEEE Control Systems Magazine, vol. 14, no. 4, pp. 25-33, August 1994.

[13] Lennart Ljung, System Identification: Theory for the User, PrenticeHall, 1986.

[14] T. Söderström and P. Stoica, System Identification, Prentice-Hall International, 1989.

[15] Shankar Sastry and Marc Bodson, Adaptive Control: Stability, Convergence, and Robustness, Prentice-Hall, Englewood Cliffs, NJ, 1989. 